\title{
Ulipristal Acetate
}

National Cancer Institute

\section{Source}

National Cancer Institute. Ulipristal Acetate. NCI Thesaurus. Code C91057.

An orally bioavailable, acetate salt of ulipristal, a selective progesterone receptor

modulator with anti-progesterone activity. Ulipristal binds to the progesterone receptor (PR), thereby inhibiting PR-mediated gene expression, and interfering with progesterone activity in the reproductive system. As a result, this agent may suppress the growth of uterine leiomyomatosis. Furthermore, by inhibiting or delaying ovulation and effecting endometrial tissue, ulipristal can be used as an emergency contraception 